\title{
Gaps in health and social care services to older adults living with HIV: a qualitative study on the perspectives of older adults and service providers
}

\begin{abstract}
Medical and social science research on older adults living with HIV (OAHIV) has shed light on the significant health needs and the many psychosocial difficulties experienced by this population. Despite the obvious need for health and social care services among OAHIV, few studies have addressed their experiences with regard to these resources. This study attempts to identify the challenges, difficulties and gaps found within social and health services intended for those located at the intersection of HIV and aging. Using a qualitative methodology, this study explores the perspectives of both older adults living with HIV (OAHIV) and their healthcare providers in order to document gaps in service delivery. Semi-structured interviews were conducted with 50 participants, including 38 OAHIV and 12 healthcare providers working with this population. Analysis was undertaken to identify themes related to challenges at individual and structural levels of practice. Several challenges were identified including; a) within the individual healthcare encounter with HIV specialists, b) accessing non-HIV related health services, and c) a lack of psychosocial resources, HIV community resources and residential resources addressing the intersection of HIV and aging. Interpreting our results through the theoretical lens of intersectionality helped identify transversal problems associated with the intersection of HIV and aging including inadequacy of resources and discrimination. Recommendations for changes to policy and practice are highlighted.
\end{abstract}

Volume 6 Issue I - 2019

\author{
Isabelle Wallach,' Shari Brotman² \\ 'Department of Sexology, Université du Québec à Montréal, \\ Canada \\ ${ }^{2}$ School of Social Work, McGill University, Canada
}

Correspondence: Isabelle Wallach, Département de Sexologie Université du Québec à Montréal, C.P. 8888, succ. Centre-ville, Montréal (QC) H3C 3P8, Canada, Tel + I I 4-987-300, Email wallach.isabelle@uqam.ca

Received: December 21, 2018 | Published: January 21, 2019

Keywords: older adults, HIV care, healthcare professional, qualitative

\section{Introduction}

The aging of people living with HIV (PLHIV), along with the rise in new HIV infections in people aged 50 and over, has attracted an increasing amount of interest among researchers and practitioners. Within both research and practice, PLHIV have been identified as an « aging » cohort beginning at the age of 50 , due to the reality of premature aging and comorbidity associated with living with HIV. Medical research on the accelerated aging of PLHIV and the multiple comorbidities associated with HIV and aging has shed light on the significant health needs of this population..$^{2-4}$ Social science research has also revealed the many psychosocial difficulties experienced by older adults living with HIV (OAHIV) ${ }^{5-9}$ Older adults living with HIV (OAHIV) are particularly vulnerable to mental health problems, ${ }^{4,10,11}$ isolation, ${ }^{5,12}$ and HIV and age-related stigma $^{1.8,12,13}$ In addition, OAHIV often find themselves confronted by a lack of emotional and instrumental support from the community due to distance from family and the absence of intimate partners. ${ }^{15,16}$ This lack of informal support results in a situation whereby OAHIV may be required to seek support from formal resources. ${ }^{2,4,16,17}$

Despite the obvious need for health and social care services among OAHIV, few studies have addressed their experiences with regard to these resources. Some authors have pointed to structural challenges related to service delivery and the inadequacy of resources addressing both HIV and aging. ${ }^{18,19}$ This inadequate response is largely blamed on a silo-effect, in which HIV-specific and ageing-specific resources

${ }^{1}$ According to the seminal works of Butler, ageism is defined as a "process of systematic stereotyping or discrimination against people because they are old". ${ }^{17}$ work in isolation from each other and have no means of organizing joint initiatives. While HIV/AIDS service organizations are often not be prepared to accommodate an aging population, and may even be openly ageist, resources intended for older people often fail to consider HIV/AIDS-related challenges resulting in a situation whereby neither sector is prepared to address complex care issues. ${ }^{18,19}$

The results of the small body of empirical research addressing HIV and aging confirm the rarity of services that specifically respond to the needs associated with this intersection. ${ }^{20-23}$ The few empirical studies that have considered difficulties experienced by OAHIV regarding their use of formal services have suggested that a primary obstacle to access involves a lack of information on existing resources. ${ }^{24}$ Other obstacles related to non-HIV-specialized health and social care services include organizational challenges, with OAHIV citing complexity and accessibility as major issues, including lengthy waiting times for service. ${ }^{24}$ The final obstacle regarding access to non-HIV-specialized services is relational, with OAHIV fearing stigmatizing attitudes of professionals towards their HIV status or to their "less acceptable" lifestyles. ${ }^{24,25}$

Some studies on the experience of service use among OAHIV have emphasized the age-related stigma found within HIV-organizations, ${ }^{16}$ along with the stigma associated with HIV within generalized health and social care services for older adults. ${ }^{24} \mathrm{~A}$ few studies make specific reference to HIV-related stigma ${ }^{8,21}$ and homophobia ${ }^{2,21}$ within residential services for older people experiencing reduced autonomy.

Studies on the difficulties experienced by OAHIV when using services reveal a lack of consideration for the intersection of HIV and aging in HIV-related services, as well as a lack of consideration for 
the interaction of various forms of stigma experienced by OAHIV due to their positioning at the intersection of several stigmatized social positions, including but not limited to age, gender, sexual orientation and class. In order to call attention to this reality, this article uses an intersectionality approach. The relevance of this approach has already been demonstrated through research on service access and the experiences of older marginalized populations, such as lesbian, gay and bisexual seniors. ${ }^{26-28}$ According to the intersectionality approach, attempts to understand the experiences of individuals should not rest upon the analysis of a single aspect of their identity. Instead, it requires consideration for how multiple social locations intersect (gender, sexual orientation, age, etc.), and result in particularized forms of structural oppression (sexism, homophobia, racism, ageism, etc.). ${ }^{29-31}$

This study attempts to identify the challenges, difficulties and gaps found within social and health services intended for those located at the intersection of HIV and aging through their own perspectives regarding these challenges, and through the perspectives of healthcare professionals that work with this population.

\section{Methods}

This research project used a qualitative approach designed to focus on uncovering the meaning people attribute to their experiences of health and social services in order to elaborate a deeper understanding of the experiences of individuals and the meaning they give to these experiences. ${ }^{3}$ The data was collected from a Montreal (Canada) medical clinic specializing in HIV/AIDS over a two-year period.

\section{Recruitment and sampling}

The sample included 38 OAHIV and 12 healthcare professionals recruited in the same HIV clinic. Inclusion criteria for OAHIV included being at least 50 years of age and living with HIV. The age of participants ranged from 50 to 73 years, 23 of whom were in their fifties and 15 of whom were aged 60 and over. In order to reflect the diversity of OAHIV subpopulations, the sample was diversified according to a variety of criteria, including gender, sexual orientation, date of diagnosis, and whether or not they had been infected through drug injection. With regard to gender and sexual diversity, the sample included 12 men who have sex with men (MSM), 14 heterosexual women and 12 heterosexual men. Furthermore, eight participants were ex-injection drug users (IDU). With regard to the date of diagnosis, 8 participants were diagnosed less than 10 years before recruitment, or at the start of the 2000s, when HIV acquired the status of chronic disease following the arrival of HAART in 1996, while the remaining thirty participants were diagnosed 10 to 25 years before recruitment. Sampling for healthcare professionals included nine physicians (three women and six men) and three nurses (one woman and two men). Among these professionals, half were aged 30 to 50 (including all nurses) and the other half were over 50. It should also be noted that half of all participants had been working in the field of HIV for 20 years or more and had therefore experienced the first years of the HIV/ AIDS epidemic.

\section{Data collection and ethical considerations}

This research was based on semi-structured individual interviews conducted in the research offices of the clinic where the participants were recruited (except for cases involving travel difficulties for OAHIV). According to ethical guidelines ${ }^{2}$, all participants were informed of the research objectives and asked to sign a consent form ahead of time to remind them of their right to not answer

${ }^{2}$ Ethical approval was received by the Research Ethics Board of McGill University (Canada) questions or to remove themselves from the project at any time. Procedures were also implemented to protect confidentiality and anonymity. The OAHIV interviews lasted an average of two and a half hours, while the professional interviews lasted an average of 1 hour. The OAHIV interviews focused on the impact of HIV and aging on various aspects of their personal and social lives, along with experiences regarding health and social care services. The central themes that were discussed with professionals included the challenges surrounding the aging of their PLHIV clientele, the specificities of providing support to OALHIV, perceptions regarding the resources currently available to OALHIV, perspectives regarding collaborative efforts with other resources, and the solutions required to improve their work and the situations of OALHIV. The interviews concluded with a sociodemographic questionnaire distributed to both groups of respondents.

\section{Data analysis}

The interviews were transcribed in full. The analysis of the data was based on a thematic analysis (Braun et Clarke, 2006), which relies on an ongoing process of comparison between the collected data and the established codes and categories. This was done using QDA Miner software. After reading the first interviews, we were able to determine the first list of categories and codes according to recurring themes identified in the responses, along with themes contained in the interview grid and literature. By regularly reviewing the material associated with each code, we were able to refine these codes and identify our categories of analysis. Both the OAHIV and professional interviews were coded separately to allow for the emergence of specific codes for each group. However, a comparison of both bodies of data allowed us to create common analytic categories. These categories primarily included "difficulties/challenges associated with HIV specialists", "difficulties associated with non-HIV-specialized health services", "challenges associated with psychosocial care", and "challenges associated with residential resources". Finally, different measures were used to validate the results, including triangulation of data sources (OAHIV and service providers), cross interpretation between and among research team members, and member checking results with both OAHIV and the clinical stakeholders that work with this population, as well as those that work within various community organizations in Quebec. ${ }^{33}$

\section{Findings}

An analysis of the responses from both providers and older adults living with HIV highlights their points of convergence, despite the slight variations that appear. The themes discussed by participants involved the challenges and difficulties associated with follow-up by HIV specialists, the problems of accessibility to non-HIV-related services, the lack of psychosocial resources targeting the intersection of HIV and aging, the gaps and inadequacies in HIV-related community resources, and the limitations of residential resources.

\section{Challenges and difficulties of follow-up by HIV specialists}

The first challenge discussed by both groups of participants involved the necessity to provide global healthcare follow-up to OAHIV due to the multiple comorbidities that afflict the population. For service providers, this involved shifting from specialized medicine to general or family medicine, as follow-up moved from a focus on the control of HIV infection to the monitoring of age-related problems. This change in practice was seen as demotivating for some practitioners: "Providing care to young people, sick but young, was very motivating, gratifying. We were in the struggle, working for the cause. And now, the 
cause turned into cholesterol and high blood pressure!" (Phys) $)^{3}$. For some other practitioners, this transition in practice was perceived as stressful and time consuming because it required further development of knowledge: "It means I have to return to studying a lot because, for many years, I only focussed on their HIV" (Phys). Although most of the older adults living with HIV interviewed appeared satisfied with medical follow-up for concerns other than HIV, some expressed disappointment toward physicians who did not give any attention to their other health problems. "Everything revolves around HIV. I tried to talk about my back a little, but... She didn't plan on that (...) There's no room for anything else." (Wom)

The second difficulty identified by both HIV specialists and older adults living with HIV involved a reduction in the time devoted to medical consultations, which limited communication. Several OAHIV noted a decrease in their physician's allotted time, as compared to their first years receiving follow-up. In discussing his HIV specialist, one participant stated: "He definitely works harder than he did, in the sense that you can tell he has a heavier patient caseload. So we can't talk as much." (IDU-HM) Physicians, for their part, deplored the lack of time they had for their older patients. They claimed it interfered with both the quality of their medical follow-up and their ability to maintain relationships of trust. "A half hour with each patient, at least, would be perfect. In general, people appreciate being there and being able to talk, not just about their health problems, but simply to say: 'I'm a grandfather. Look at this photo.." (Phys) According to these professionals, the time aspect appeared all the more crucial for OAHIV because this population was often seen as isolated and seeking psychological support from their treating physician.

While less common, the reality of ageism was indicated by several participants. Some physicians recognized their occasional irritation with certain OAHIV (due to their slowness, for example), which they attributed to aging. "The older ones, they take a long time to change (...) They take off their shirt... [mimicking the slow gestures] Oh, my goodness! You know, that's the older clientele. They're less mobile, they have more osteoarthritis. " (Phys) Although less common, ageist attitudes were reported by a few OAHIV. One participant stated that her physician finds her more of a "hypochondriac with age." Another MSM participant mentioned an experience of ageist prejudice on the part of his physician regarding his intimate life. "She said: 'You fifty-somethings are always complaining! You're all the same! You all want to look younger so you can keep seducing (...) And you're frustrated!'(MSM)

\section{Access difficulties for non-HIV-related health services}

The main issue that emerged from the responses of participants with regard to non-HIV-related health services involved access difficulties. Both professionals and OAHIV reported a preoccupation with long waiting times across the health network, whether it involved access to medical examinations or scheduling medical consultations with specialists. This challenge was made all the more problematic because OAHIV suffer from a number of health issues associated with the interaction of HIV and aging, or with the undesirable effects of antiretroviral medication. Physicians who attempted to schedule their own medical appointments for patients struggled with the same difficulties, as waiting times threatened the vital prognosis of OAHIV. "I lost a patient to lung cancer because he was never seen. It took nine

${ }^{3}$ The abbreviations that follow quotations correspond to the following code: Wom=woman; IDU-W=female injection drug user or former injection drug user; $\mathrm{HM}=$ heterosexual man; IDU-HM=heterosexual male injection drug user or former injection drug user; $\mathrm{MSM}=$ man who has sex with men; Phys=physician; Nur=nurse month to see him before the investigation could start. I tried to make phone calls but nothing came of it." (Phys)

The second problem, widely reported by OAHIV participants and professionals alike, involved HIV-related stigma that hinders access to certain health services. Both cohorts identified specialists and paramedical professionals as often reticent to treat OAHIV. "As soon as they arrive and identify their seropositivity, the doors close a little (...) If they're constantly being transferred to another waiting list, something clearly isn't working." (Phys)

Several OAHIV reported differential treatment on the part of healthcare professionals due to their infection status. "In the emergency room, they tend to isolate you into a corner and forget about you (...) A lot of people [with HIV] go through that." (Wom) In some cases, HIV-related stigma by non-HIV-specialized healthcare professionals extended to refusing to provide care. I started getting something on my penis, like a gash, so he [my HIV specialist] sent me to a specialist. He touched me for a few seconds: 'Ah, you're fine! You're fine, go away!' I could tell I was bothering him. But when I spoke to another specialist, he sent me for x-rays." (MSM)

Finally, and in addition to HIV-related stigma, certain participants reported negative attitudes toward their status as IDU. In this situation, participants related that problems involving waiting times and refusal to provide care were due to the stigma associated with drug addiction. "When you're catalogued like I am, you're a society leftover. You can feel it. You can see it (...) I had to meet with three surgeons before one of them agreed to take care of me." (HM-IDU)

\section{Lack of HIV and age-related psychosocial resources}

Service providers and OAHIV agreed about the lack of psychosocial services available to address HIV and age-related needs. "Support from a social worker, that's what's missing. There aren't any for the HIVs. Our patients are going through difficult times, and there's no support." (Phys) "Many of them suffer alone at home, anxious, depressed (...) Some of them would need a lot more psychological monitoring... I've met several who had been left to themselves." (MSM)

Several professionals also highlighted the problematic silo that exists pointing to HIV-specialized and generalized psychosocial services. While the HIV-specialized structures were reported to fail to consider aging as a problem, generalized services failed to provide a suitable response to the psychological difficulties associated with HIV. "the STBBI [sexually transmissible and blood-borne infections] psychological services counter, they only want people who are having trouble with the diagnosis, or the antiretrovirals. Then the problem of aging (...) they don't think it's an HIV-related problem, so we have to send them to mainstream services where they don't have the expertise or adaptability required for this [HIV]." (Phys)

\section{Insufficient and inadequate HIV community resources}

Practitioners and OAHIV agreed that the community sector did not provide a sufficient response regarding the needs of OAHIV. Participants confirmed that a shortage of community organizations specializing in HIV, brought on by years of federal budget cuts throughout the sector, contributed to this situation. "Before, we had a committee of people living with HIV, right here (...) It no longer exists. It closed down... Unfortunately. Because, here in Montreal, it was the only committee that offered a lot of help." (HM) "There are fewer groups and fewer services available to them (...) The community sector used to have more presence." (Phys) 
While the reduction of community resources concerned all PLHIV, the trend was considered especially alarming for OAHIV, because their numbers are on the rise. "There are more and more [people 50 and over] because people used to die very early ten or so years ago. But today, they live longer (...) There will certainly be fewer and fewer community resources as the demand increases." (Nur)

Beyond the shortage of community organizations, existing inadequacies within organizations was also mentioned by participants. Several OAHIV deplored the absence of programs and resources that specifically target the 50 and over population. In fact, many among them mentioned the gap that often exists between their concerns and those of younger people. They believed they would have more to gain from sharing their experiences with people in their own age group but had little opportunity to do so. "Whether we like it or not, the twenty to fifty group, it doesn't work. Our needs are too different. We have different lifestyles." (Wom) Responses from participants also highlighted the need for programs to expand their scope beyond psychological support and discussion groups to include socialization in order to help them overcome their social and intimate isolation. "There really aren't many resources for [older] gay men who come from bars or the gay scene. What those men also want is a way to meet each other. Not sure there are many." (Phys) "That's what I was looking for. If there was an organization for men and women aged 50 and over where we could meet, maybe I could find a partner." (HM)

\section{Non-existent or unsuitable residential resources}

Participant responses revealed their fears regarding future housing possibilities for OAHIV with a loss of autonomy. Professionals and OAHIV agreed on the absence of residential resources intended for OAHIV, even though their needs and numbers will likely increase in the coming years. For example, one participant talked about the fact that palliative care centres are not adapted to an aging population with HIV. "In the best of worlds, we would have more housing options. Not just for end-of-life, but for older, seropositive people." (Phys)

Similarly, OAHIV worried about their future and wondered where they will spend their final years, especially in the face of loss of autonomy. "The CHSLD [long term care residence] is for older people. Will there be a hospital for older people living with HIV? What's the future like? It's not reassuring." (IDU-W) Furthermore, some insisted on the necessity of adequate medical support, along with personnel that are trained to handle the specific medical needs associated with HIV and aging within long term care services.

A lack of resources that specifically target the OAHIV population raised another concern regarding the risk of experiencing stigma within senior's residences and long-term care facilities from both residents and caregivers alike. "That's what I'm afraid of. When you get older, they'll stick you in a corner because you have HIV, and the girl won't want to wash you because you have HIV." (HM) Both groups of participants also mentioned the risk that HIV-related stigma can intersect with homophobia. "You go to a retirement home, and you have to hide the fact that you're a homosexual (...) Sometimes, it takes a long time to come out of the closet. You don't want to have to get back in when you're older." (MSM)

\section{Discussion}

The findings from this study identify the many challenges and gaps that exist within services designed to respond to HIV and agerelated needs. The results shed light on the inadequacies that exist within services available to OAHIV, along with the lack of resources specifically geared toward this clientele. Beyond the validation of empirical data and the challenges addressed by other studies ${ }^{20,22,23}$ interpreting our results according to the theoretical intersectionality framework helped identify three transversal problems across all formal resources. These are: 1) an inadequacy of resources to respond to the needs associated with HIV and aging, 2) structural problems within the health and social care service system which are amplified for OAHIV, and 3) the multiple forms of discrimination experienced by OAHIV due to their position at the intersection of HIV and aging. For OAHIV, these three types of problems will often interact and manifest simultaneously.

Firstly, the inadequacy of services when responding to the intersection of HIV and aging was considered a central factor in the discourse of both OAHIV and practitioners interviewed for this study. With regard to HIV physicians, the obligation to shift from a practice exclusively focussed on HIV to one which is more generalized is experienced negatively, whether that be because of a lack of interest or of adequate knowledge. This reality is partially explained by the specialization of practitioners, with those who had been trained specifically in the field of infectious disease reporting less discomfort with respect to treatment of comorbidities related to age, than those who had been trained in general medicine. This reality is supported by the literature. ${ }^{22}$

The impact of silos identified by several authors. ${ }^{18,19}$ has been confirmed by our study as one of the sources of resource inadequacy. Within psychosocial services, the inability to respond to the difficulties associated with the intersection of HIV and aging might stem from the organization of the health system, which distinguishes psychological services offered to the general population, including older people, from those intended for PLHIV. A significant lack of consideration for aging is also present within community organizations associated with HIV and creates shortcomings, such as a lack of programs intended exclusively for an older clientele, or programs that respond to unique needs, such as overcoming isolation. Finally, participants identified the inadequacy of residential services that are limited to palliative care services within the HIV sector and do not take into account the need for housing for OAHIV, who may live for many years, with or without a loss of autonomy.

The second source of difficulties identified involves structural problems within the health system which appear to have significant repercussions for the OAHIV population. Firstly, the limited duration of medical consultations is seen as a problem that dramatically affects OAHIV due to their isolation and significant need for psychological support, considering that physicians often constitute an essential resource in this regard. Difficulties in accessing specialized services creates particularly harmful repercussions for OAHIV due to their significant health needs, particularly for those experiencing multiple comorbidities. ${ }^{2-4}$ Similarly, the lack of psychological resources, is particularly detrimental to OAHIV due to the prevalence of mental health problems within this population. ${ }^{4,10,11}$ Finally, the reduction in HIV community resources, has a significant impact on older adults, particularly considering the significant rise in the number of people aged 50 and over diagnosed with this condition.

The third difficulty facing OAHIV involves multiple forms of discrimination within various types of social and health services due to their positioning at the intersection of several categories of oppression (HIV, old age, drug use, sexual minority status). Several studies have documented the interplay between ageism in HIV services and HIVrelated stigma within generalized and gerontological social and health 
services, including residential care. ${ }^{8,21,24}$ Our research has highlighted some of the ageist behaviours exhibited by healthcare professionals specializing in HIV as well as discriminatory attitudes towards HIV within general health services. For IDUs specifically, discrimination regarding past or present injection drug use within general health services, as well as the risk of experiencing HIV-related stigma in senior's residences, may intersect with other forms of stigma, such as homophobia.

This research presents several limits. The main one involves the recruitment of participants, all of whom were selected from the same medical clinic specializing in HIV. Different responses might have emerged had the recruitment been conducted across several HIV-specialized sites such as community organizations, or across geographical contexts such as small-town or rural locations. Furthermore, while our research was based on responses from fifty participants, the number of healthcare professionals interviewed, along with the low sampling diversification of professionals (physicians and nurses), remains small and does not allow for the generalization of results. It would be worthwhile if future studies were to include stakeholders from various sectors and professions like psychology, social work, community organizations, and senior's residences. ${ }^{34-38}$

\section{Conclusion}

Service providers have an important role to play in challenging ageist stereotypes and encouraging discussion about ageing within HIV-related services and HIV in ageing services. Organizations can support practitioners in doing so by adequately preparing them to address a topic that is difficult for service providers to address. Considering that service providers and OAHIV mentioned the importance of having time to develop rapport and trust, creating opportunities for this occur is essential. Since restrictions on time are significant for physicians, counseling initiatives might be better placed in the hands of community nurses and other allied health professionals. Ensuring the availability of longer-term counselling would be a challenging undertaking, particularly in the current climate of scarcity within public sector health care and social services. Support programs, as well as community follow-up are other services that organizations can build to ensure that there is opportunity to address ageing with HIV. Specific attention to issues facing people with ageing-related concerns (such as dementia and other cognitive impairments) is essential given the unique experiences and concerns of this population. Counselling and educational initiatives targeting OAHIV should involve addressing issues such as internalized stigma, self-esteem and identity and social isolation.

Training for service providers would be beneficial in promoting knowledge and addressing discomfort in line with multiple stigma related to HIV and aging. Finally, policy initiatives designed to build expertise across systems and specialties and that supports the development of intersectional services must be made a priority. This includes the development of interdisciplinary teams that can be accessed through both HIV and ageing services, and within residential settings, in order to ensure that responses are designed with the needs and realities of OAHIV in mind. Only then will stigma resulting from intersectional forms of discrimination in health care be reduced and the particular medical and psychosocial concerns of OAHIV be addressed.

\section{Funding details}

This project was supported through a research grant from the Quebec Ministry of Families and Seniors (program: Soutien aux initiatives visant le respect des aînés (SIRA)).

\section{Acknowledgments}

The authors would like to thank Xuân Ducandas and Michel Martel for their assistance with data collection and analysis, and all the people who participated for their trust and availability.

\section{Conflicts of interest}

Authors declare that there is no conflicts of interest.

\section{References}

1. Blanco JR, Jarrín I, Vallejo M, et al. Definition of Advanced Age in HIV Infection: Looking for an Age Cut-Off. Aids Research and Human Retroviruses. 2012;28(9):800-806.

2. Cahill S, Valadéz R. Growing Older with HIV/AIDS: New Public Health Challenges. American Journal of Public Health. 2013;103(3):e7-e15.

3. High KP, Brennan-Ing M, Clifford DB, et al. HIV and aging: state of knowledge and areas of critical need for research: A report to the NIH Office of AIDS Research by the HIV and Aging Working Group. Journal of Acquired Immune Deficiency Syndromes. 2012;60(1):S1-S18.

4. Brennan-Ing M, Seidel L, London A, et al. Service utilization among older adults with HIV: the joint association of sexual identity and gender. Journal of Homosexuality. 2014;61(1):166-196.

5. Brennan M, Karpiak SE, Shippy A, et al. Older Adults with HIV: An In-Depth Examination of an Emerging Population. USA: Nova Science Publishers; 2009. 77 p.

6. Roger KS, Mignone J, Kirkland S. Social aspects of HIV/AIDS and aging: a thematic review. Canadian Journal on Aging/La Revue canadienne $d u$ vieillissement. 2013;32(3):298-306.

7. Sankar A, Nevedal A, Neufeld S, et al. What do we know about older adults and HIV? A review of social and behavioral literature. AIDS Care. 2011;23(10):1187-1207.

8. Wallach I, Brotman S. Ageing with HIV/AIDS: a scoping study among people aged 50 and over living in Quebec. Ageing and Society. 2013;33(7):1212-1242.

9. Wallach I, Brotman S. The intimate lives of older adults living with HIV: A qualitative study of the challenges associated with the intersection of HIV and ageing. Ageing and Society. 2017;38(12):2490-2518.

10. Grov C, Golub SA, Parsons JT, et al. Loneliness and HIV-related stigma explain depression among older HIV-positive adults. AIDS care. 2010;22(5):630-639.

11. Kalichman SC, Heckman T, Kochman A, et al. Depression and Thoughts of Suicide Among Middle-Aged and Older Persons Living With HIVAIDS. Psychiatric Services. 2000;51(7):903-907.

12. Emlet CA. An Examination of the Social Networks and Social Isolation in Older and Younger Adults Living with HIV/AIDS. Health \& Social Work. 2006;31(4):299-308.

13. Emlet CA. Experiences of stigma in older adults living with HIV/ AIDS: a mixed-methods analysis. AIDS Patient Care and Studies. 2007;21(10):740-750.

14. Emlet CA. You're awfully old to have this disease: Experiences of stigma and ageism in adults 50 years and older living with HIV/AIDS. The Gerontologist. 2006;46(6):781-790.

15. Cantor MH, Brennan M, Karpiak SE. The Social Support Networks of Older People with HIV. 2009.

16. Shippy RA, Karpiak SE. Perceptions of Support Among Older Adults with HIV. Research on Aging. 2005;27(3):290-306.

17. Lagacé M. Lâgisme: Comprendre et changer le regard social sur le vieillissement. Québec, Qc: Presses de 1 Université Laval; 2010. 298 p. 
18. Emlet CA, Poindexter CC. Unserved, Unseen, and Unheard: Integrating Programs for HIV-Infected and HIV-Affected Older Adults. Health and Social Work. 2004;29(2):86-96.

19. Linsk NL, Fowler JP, Klein SJ. HIV/AIDS Prevention and Care Services and Services for the Aging: Bridging the Gap Between Service Systems to Assist Older People. Journal of Acquired Immune Deficiency Syndromes. 2003;33(2):S243-S250.

20. Fritsch T. HIV/AIDS and the Older Adult: An Exploratory Study of the Age-Related Differences in Access to Medical and Social Services. Journal of Applied Gerontology. 2005;24(1):35-54.

21. Furlotte C, Schwartz K, Koornstra JJ, et al. 'Got a room for me?'Housing Experiences of Older Adults Living with HIV/AIDS in Ottawa. Canadian Journal on Aging/La Revue canadienne du vieillissement. 2012;31(1):37-48

22. Cresswell FV, Levett T. Specialist care of older adults with HIV infection in the UK: a service evaluation. HIV Medicine. 2017;18(7):519-524.

23. Seidel L, Karpiak S, Brennan-Ing M. Training senior service providers about HIV and aging: evaluation of a multiyear, multicity initiative. Gerontology \& Geriatric Education. 2017;38(2):188-203.

24. Brennan M, Karpiak SE, London AS, et al. A Needs Assessment of Older GMHC Clients Living With HIV. USA: Aids Community Research Initiative of America (ACRIA); 2010. 50 p.

25. Emlet CA. Knowledge and Use of AIDS and Aging Services by Older, HIV-Infected Adults. Journal of HIV/AIDS \& Social Services. 2004;3(1):9-24

26. Brotman S, Ferrer I, Sussman T, et al. cces and equity in the design and delivery of health and social care of LGBTQ older adults: A canadian perspective. In: Orel NA, Fruhauf CA, editors. The Lives of LGBT Older Adults: Understanding Challenges and Resilience. USA: American Psychological Association; 2015. p. 111-140.

27. Brotman S, Ryan B, Collins S, et al. Coming Out to Care: Caregivers of Gay and Lesbian Seniors in Canada. The Gerontologist. 2007;47(4) 490-503.
28. Brotman S, Ryan B, Cormier R. The health and social service needs of gay and lesbian elders and their families in Canada. The Gerontologist. 2003;43(2):192-202.

29. Crenshaw K. Mapping the margins: intersectionality, identity politics, and violence against women of color. In: Crenshaw K, Gotanda N, editors. Critical Race Theory: The Key Writings That Formed the Movement. USA: The New Press; 1995. p. 357-383.

30. Collins P. Black Feminist Thought: Knowledge, Consciousness, and the Politics of Empowerment. USA: Routledge; 2000.

31. Hancock AM. Intersectionality as a normative and empirical paradigm Politics \& Gender. 2007;3(2):248-254.

32. Creswell JW. Qualitative Inquiry and Research Design: Choosing Among Five Approaches. 3rd ed. Thousand Oaks, CA: SAGE Publications; 2012.

33. Creswell JW, Miller DL. Determining validity in qualitative inquiry. The ory Into Practice. 2000;39(3):124-130.

34. Braun V, Clarke V. Using thematic analysis in psychology. Qualitative Research in Psychology. 2006;3(2):77-101.

35. Brennan SE, Karpiak RA, Shippy, et al. Older Adults with HIV: An InDepth Examination of Emerging Population. USA: Nova Science Publishers; 2009. p. 61-74.

36. Grenier A, Brotman S. Les multiples vieillissements et leurs représentations. In : Charpentier M, Guberman N, edtiors. Vieillir au Pluriel : Perspectives Sociales. Québec, Qc: Presses de 1'Université du Québec. 2010. p. 23-54.

37. Shippy RA, Karpiak SE. The aging HIV/AIDS population: Fragile social networks. Ageing and Mental Health. 2005;9(3):246-254.

38. Calasanti T, Slevin K. Age Matters: Re-Aligning Feminist Thinking. USA: Routledge; 2006. 